\title{
Evidence first, practice second in arthroscopic surgery: use of placebo surgery in randomised controlled trial
}

\author{
Kazuha Kizaki, ${ }^{1,2}$ Lisa J Schwartz, ${ }^{1,3}$ Olufemi R Ayeni (i) 1,2
}

${ }^{1}$ Department of Health Research Methods, Evidence, and Impact, McMaster University, Hamilton, Ontario, Canada

${ }^{2}$ Division of Orthopaedic Surgery, McMaster University, Hamilton, Ontario, Canada ${ }^{3}$ Department of Clinical Epidemiology and Biostatistics, McMaster University, Hamilton, Ontario, Canada

Correspondence to Dr Olufemi R Ayeni, Division of Orthopaedic Surgery, McMaster University, Hamilton, ON

L8N3Z5, Canada;

femiayeni@gmail.com

Received 27 May 2019 Revised 19 September 2019 Accepted 24 October 2019 Published Online First

2 November 2019

\section{ABSTRACT}

The application of evidence-based medicine helps clinicians avoid unnecessary procedures and decreases unnecessary harm for future patients while sparing economic burdens. Randomised controlled trials (RCTs) most accurately produce best research evidence. In arthroscopic surgery, however, many procedures have been extensively used without supportive evidence verified with RCTs. In this paper, we introduce two procedures (arthroscopic partial menisectomy for degenerative knees and arthroscopic subacromial decompression for subacromial pain syndrome), where over 30 years of procedure usage has continued prior to garnering evidence for the inefficacy of the procedures. The situations are attributed to the fact that clinical trials in arthroscopic surgeries are challenging given the use of placebo controls. A placebo-control RCT can accurately answer research questions about efficacy and safety of surgical procedures; however, the majority of arthroscopic surgeries in practice have not been rigorously tested against placebo surgeries. This is because preparing surgical placebo controls, known as sham surgeries, are ethically controversial. Also considering that high-quality study results often do not change clinical practice due to insufficient knowledge translation, the benefits of such trials may be uncertain to society at large. Additionally, there are a lack of clear guidelines for conducting arthroscopic placebo surgeries in RCTs. We hope that this article helps drive discussion about appropriate use of placebo surgeries in RCTs to produce the best quality evidence in arthroscopic surgery.

\section{INTRODUCTION: EVIDENCE-BASED MEDICINE}

Almost 30 years have passed since David Sackett and Gordon Guyatt first introduced evidence-based medicine (EBM). EBM is the conscientious use of current best evidence in making decisions about patient care. EBM also includes the integration of best research evidence with clinical expertise and patient values in the decision-making process for patient care. ${ }^{1}$ Clinical expertise refers to the clinician's accumulated experience, education and clinical skills. The patient brings their own personal preferences and unique concerns, expectations and values to the encounter. Best research evidence is the accumulation of reliable studies with sound research methodology, consisting of case reports/ series, observational studies (ie, cross-sectional, cohort and case-control studies) and experimental studies. Experimental studies, namely randomised controlled trials (RCTs), most accurately answer research questions and play a prominent role in best research evidence. ${ }^{2}$ The best research evidence helps clinicians avoid unnecessary procedures and decreases unnecessary harm for future patients while also sparing the economic burden.

\section{Practice first, evidence second in arthroscopic surgery}

In cancer treatment, a new chemotherapy is investigated in multilevels, laboratory and phase I-III studies, for efficacy and safety. ${ }^{3}$ Before it is to be used in clinical practice, it is examined for safety and biological rationale. Afterwards, it can be used in clinical practice, and all patients are followed in a registry for observing adverse events and clinical effects. This framework is strictly monitored and controlled.

In arthroscopic surgery, by comparison, many procedures have been extensively used without supportive evidence. Arthroscopic partial menisectomy was first introduced in 1970s and the procedure has been globally adopted. Approximately 700000 arthroscopic partial menisectomies were performed in 2006 in the USA, resulting in an estimated US\$4 billion in medical costs. ${ }^{4}$ However, RCTs using placebo surgery demonstrated that arthroscopic partial menisectomy did not improve functional outcomes for degenerative meniscus tear. $^{5}{ }^{6}$ In 2017, a clinical practice guideline for degenerative meniscal tear recommended against arthroscopic knee surgery. ${ }^{7}$ It has taken almost 40 years to test the efficacy of the procedure. Similarly, in the 1980s, arthroscopic subacromial decompression surgery for subacromial pain syndrome was first introduced. This procedure also spread globally, with 21355 procedures performed in 2009 and 2010 in England alone. ${ }^{8}$ In 2018, two RCTs using placebo surgery showed that arthroscopic subacromial decompression provided no benefit over placebo surgeries for improving pain, function and quality of life. ${ }^{9}{ }^{10}$ In 2019 , a clinical practice guideline recommended that subacromial decompression surgery should not be offered to patients with subacromial pain syndrome. ${ }^{11}$ In these cases, over 30 years of procedure usage has continued prior to garnering evidence for the inefficacy of the procedures.

\section{Why practice first, evidence second in arthroscopic surgery}

As compared with other medical fields, arthroscopic surgery has two distinct characteristics. First, arthroscopic surgery is minimally invasive with key hole skin incisions and risk of the procedure is relatively small. The predominant complication is risk of infection, although the prevalence is very low with $\sim 0.2 \%$. $^{12}$ Due to this minimal risk and potential upside in improved symptoms, procedures in arthroscopic surgery are growing in prevalence. 
Second, clinical studies in arthroscopic surgery are difficulty to conduct using placebo controls. A double-blinded placebocontrol RCT can answer research questions about efficacy and safety of surgical procedures; however, the majority of surgical interventions in surgical practice have not been based on RCTcertified evidence. ${ }^{13}$ Preparing surgical placebo controls, known as sham surgeries, are ethically controversial. Also, there are a lack of clear guidelines for conducting arthroscopic placebo surgeries in RCTs.

\section{Ethical issues in arthroscopic placebo surgery}

Three ethical arguments have been put forth against the use of placebo surgery: unnecessary harm, beneficiaries of placebo surgery and active deceptiveness. ${ }^{14}$ The most prominent issue for participants is unnecessary harm with placebo surgeries. In 1947, the Nuremberg code released a landmark document in clinical research ethics stating that all research should avoid any unnecessary physical or mental suffering. ${ }^{15}$ Skin incisions in arthroscopic placebo surgery can be interpreted as an unnecessary suffering. Another debate is who benefits for RCTs using placebo surgeries. RCTs using placebo surgeries most accurately answer research questions and benefit society and future patients; however, it may not be beneficial for participants for themselves. It is potentially unethical in reference to the Helsinki declaration (1964), which specifies that concern for the interests of the subject must always prevail over the interests of society and science. ${ }^{16}$ Thus, in cancer treatment studies, for example, particularly in phase II or III cancer drug trials, it is not permitted to include patients in placebo trials where they are only at risk of an intervention's adverse effects with no potential for individual benefit from receiving the existing standard of treatment. The third concern is active deceptiveness. Surgeons have to deceive participants in order to keep blinding of participants; however, it violates the principle of respect for persons and their autonomy for decision-making. Also, deceptiveness can eventually jeopardise doctor-patient relationships.

We introduce counterarguments against these concerns. First, an arthroscopic placebo surgery gains more information as a diagnostic tool to evaluate the intra-articular condition and in this regard, a placebo surgery with skin incisions is not an unnecessary harm. Based on current evidence, arthroscopic diagnosis is more accurate compared with diagnostic imaging, such as MRI. Second, participants who are assigned to the arthroscopic placebo surgery group can still receive benefits due to placebo effect. The placebo effect is a psychological effect and it does not produce any physiological changes; however, it significantly helps improve clinical outcomes. In a systematic review examining the impact of the placebo effect, a large clinical effect with effect size (Cohen's d) $>0.8$ from baseline has been observed in the placebo surgery group. ${ }^{17}$ The results show that participants also receive benefits from RCTs using arthroscopic placebo surgery (such as those associated with placebo-effect), in addition to gaining new scientific knowledge for society and future patients.

For issues with deceptiveness in RCT, succinct informed consent can prevent a violation of participants' autonomy and decrease participants' misconception. Before enrolling in the study, the possibility of participants' being assigned to the placebo-control group and being blinded from the allocation should be explained. Also, participants should be informed that the aim of the study is to gain new insights for a true state of uncertainty (clinical equipoise), and not to provide therapeutic treatment for participants. It may be preferable to tell participants that they will be monitored with an additional layer of safety to comprehensively evaluate their well-being throughout the study process. This is a potential benefit for participants.

Despite this, a noticeable issue still exists regarding the potential benefit of RCTs using arthroscopic placebo surgery, namely difficulty of uptake

from knowledge translation of study results in arthroscopic surgery. For example, arthroscopic partial menisectomy is ineffective for degenerative knee conditions, but many arthroscopic partial menisectomy procedures are still being conducted in the setting of degenerative knees. ${ }^{18}$ Unfortunately, as seen in this case, study results even in RCTs using placebo surgery are not readily or speedily adopted in clinical practice. Thus, in consideration of the slow uptake and ambiguity of application of study results in clinical practice, it may be ethically unacceptable for participants to be at risk of unnecessary harm (placebo surgery) for uncertain benefit to society and future patients.

This current situation arises from insufficient knowledge translation. Researchers tend to neglect solutions to deliver new, highquality evidence-based information to patients. ${ }^{19}$ In arthroscopic surgery, many research projects have been launched for surgical innovation, whereas only a few papers exist investigating knowledge translation in arthroscopy. Pronovost et al described an integrated approach to enhance knowledge translation with four steps: first, summarise the evidence for interventions to improve a specific outcome; second, identify local barriers to implementation; third, measure performance, and fourth, ensure all patients reliably receive the intervention. ${ }^{19}$ In the case with arthroscopic partial menisectomy for degenerative knees, research is currently in the second stage (identification of local barriers to implementation). We are in need of investigations into nonadherence with the new guideline suggesting the avoidance of arthroscopic partial menisectomy for degenerative knees. Some surgeons do not recognise that the adherence of EBM guidelines can help minimise unnecessary harm, and would be the most ethically applicable approach. In this case, avoidance of a traditional method may seem like a difficult change in practice, but will reduce harm in patients who can avoid unnecessary surgery. ${ }^{20}$

As a first step to break the local barrier and move forward to adopt study results into clinical practice, we recommend sending a message of the importance of evidence first, and procedure second in arthroscopic surgery. A new arthroscopic procedure should be adopted in clinical practice after the verification of the efficacy of the procedure through best research evidence (evidence first). Also, a conceptual framework for dissemination of new evidence-based treatment guideline in arthroscopic surgeries should be constructed in collaboration with stakeholders and policymakers in various fields, such as patient education, organisational learning and social marketing in order to supply ethically desirable treatments for patients. ${ }^{21}$ High adaptability of high-quality study results into clinical practice would make it ethically applicable to recruit patients into RCTs using placebo surgery. The current controversy about the use of arthroscopic placebo surgery lies in the fact that patients in the placebo-arm in RCTs are at risk with few individual benefit (potentially beneficial in light of arthroscopic diagnosis and placebo effect) and uncertain future population benefit.

\section{Proposals for RCT with arthroscopic placebo surgery}

We appreciate the superiority of RCT arising from randomisation, which balances unknown and known confounding, and prognostic factors between intervention and control groups, to most accurately examine therapeutic effects of intervention. However, using RCTs using arthroscopic placebo surgeries is not typical because there are no clear ethical guidelines. We introduce two 
articles (Bunzli et al and Savulescu et al) proposing prerequisites for surgical RCTs using placebo surgeries. ${ }^{22} 23$

Bunzli et al summarised recommendation for use of placebo surgery in American Medical Association council on Ethical and Judicial Affairs with six domains: previous studies, informed consent, major modification, placebo effect, risk evaluation and alternative treatment.

1. Sham controls should only be used when no other trial design will yield the same data.

2. Careful attention is paid to the informed consent process when enrolling participants.

3. Sham controls are not justified when testing the effectiveness of a minor modification to an existing procedure.

4. Sham controls may be justified if it is known that the disease being studied is susceptible to a placebo effect.

5. The risks of the sham-control operation should be relatively small.

6. The patient must be offered the opportunity to receive the standard treatment if it is found to be efficacious at the end of the study.

In previous studies, the presumable efficacy and adverse effects should be evaluated a priori in non-RCT studies before preparing an RCT. In obtaining informed consent, active deceptiveness is essential to ensure the quality of evidence in RCT. However, it risks harming a participant's autonomy. The patients should be informed enough to consent to the possibility of being assigned to the placebo surgery-control group and being blinded for the duration of the study. Preparing placebo surgery control should be considered only for a new surgical treatment which potentially guides a major modification in treatment guideline. The placebo effect is noticeably enhanced when participants expect a greater benefit over the potential risk. Arthroscopic surgeries are likely to produce a greater placebo effect owing to relatively small risks (key hole, minimally invasive wounds). In terms of risk evaluation, the risks associated with arthroscopic placebo surgeries should be minimised. The potential adverse events should be curable if they happen. Participants should also have a right to stop participation in RCTs at any time and be able to receive an alternative treatment based on their own decision (autonomy).

Savulescu et al also proposed that surgical randomised placebo-controlled trials are ethically applicable if the following conditions are fulfilled: clinical equipoise, major modification, risk evaluation, no deception, previous studies and additional benefit:

1. The presence of equipoise, defined as a lack of unbiased evidence for efficacy of an intervention

2. Clinically important research question

3. The risk to participants is minimised and reasonable

4. There is uncertainty about treatment allocation rather than deception

5. There is preliminary evidence for efficacy, which justifies a placebo-controlled design

6. Ideally, the placebo procedure should have some direct benefit to the patient, for example, as a diagnostic tool.

Both proposals suggested the importance of previous studies in which a new surgical procedure should be evaluated a priori for risk and benefit, and also, it should be modified enough to potentially change the treatment guidelines. They additionally advocated the necessity of properly informed consent with respect for participants' autonomy. To retain good doctor-patient relationships, the possibility of actively being deceived should be specified in obtaining informed consent.

These proposals help create a conceptual framework for preparing arthroscopic placebo surgery in RCT. Based on the history of evidence for arthroscopic partial menisectomy for degenerative knees and arthroscopic subacromial decompression for subacromial pain syndrome, we are urged to evaluate the evidence in arthroscopic surgery preparing RCT using arthroscopic placebo surgery.

\section{CONCLUSION}

This is an article to address the necessity of RCTs using arthroscopic placebo surgeries. In arthroscopic surgery, practice has preceded evidence for two reasons: relatively small risk with key hole skin incisions, and no clear guidelines for preparing arthroscopic placebo surgeries. We introduced several ethical principles for consideration regarding the use of placebo surgery in arthroscopy: previous studies, clinical equipoise, risk evaluation, major modification, informed consent, alternative treatment and placebo effect. We hope that this article helps drive discussion for RCT using placebo surgery to produce the best quality evidence in arthroscopic surgery.

Acknowledgements We acknowledge Andrew Duong for constructive discussion and proofing assistance.

Contributors KK and ORA conceptualised this research project. KK wrote the paper. LJS critically reviewed the ethical issues. ORA supervised this research project.

Funding The authors have not declared a specific grant for this research from any funding agency in the public, commercial or not-for-profit sectors.

Competing interests None declared.

Patient consent for publication Not required.

Provenance and peer review Not commissioned; externally peer reviewed.

Data availability statement All data relevant to the study are included in the article

\section{ORCID iD}

Olufemi R Ayeni http://orcid.org/0000-0003-4219-8564

\section{REFERENCES}

1 Sackett DL, Rosenberg WM, Gray JA, et al. Evidence based medicine: what it is and what it isn't. BMJ 1996:312(7023):71-2.

2 Lohmander LS, Roos EM. The evidence base for orthopaedics and sports medicine: scandalously poor in parts. Br J Sports Med 2016;50(9):564-5.

3 Sridhara $R$, Johnson JR, Justice $R$, et al. Review of oncology and hematology drug product approvals at the US food and drug administration between July 2005 and December 2007. J Natl Cancer Inst 2010:102(4):230-43.

4 Cullen KA, Hall MJ, Golosinskiy A. Ambulatory surgery in the United States, 2006. Natl Health Stat Report 2009;28:1-25.

5 Moseley JB, O'Malley K, Petersen NJ, et al. A controlled trial of arthroscopic surgery for osteoarthritis of the knee. N Engl J Med 2002;347(2):81-8.

6 Sihvonen R, Paavola M, Malmivaara A, et al. Arthroscopic partial meniscectomy versus sham surgery for a degenerative meniscal tear. N Engl J Med 2013;369(26):2515-24

7 Siemieniuk RAC, Harris IA, Agoritsas T, et al. Arthroscopic surgery for degenerative knee arthritis and meniscal tears: a clinical practice guideline. BMJ 2017;10.

8 Judge A, Murphy RJ, Maxwell R, et al. Temporal trends and geographical variation in the use of subacromial decompression and rotator cuff repair of the shoulder in England. Bone Joint J 2014;96-B(1):70-4.

9 Beard DJ, Rees JL, Cook JA, et al. Arthroscopic subacromial decompression for subacromial shoulder pain (CSAW): a multicentre, pragmatic, parallel group, placebo-controlled, three-group, randomised surgical trial. The Lancet 2018;391(10118):329-38.

10 Paavola M, Malmivaara A, Taimela S, et al. Subacromial decompression versus diagnostic arthroscopy for shoulder impingement: randomised, placebo surgery controlled clinical trial. BMJ 2018;19.

11 Vandvik PO, Lähdeoja T, Ardern C, et al. Subacromial decompression surgery for adults with shoulder pain: a clinical practice guideline. BMJ 2019;55(1).

12 Brignardello-Petersen R, Guyatt GH, Buchbinder R, et al. Knee arthroscopy versus conservative management in patients with degenerative knee disease: a systematic review. BMJ Open 2017;7(5):e016114.

13 Lim HC, Adie S, Naylor JM, et al. Randomised trial support for orthopaedic surgical procedures. PLoS One 2014;9(6):e96745.

14 Niemansburg SL, van Delden JJM, Dhert WJA, et al. Reconsidering the ethics of sham interventions in an era of emerging technologies. Surgery 2015;157(4):801-10.

15 Ghooi R. The Nuremberg Code-A critique. Perspect Clin Res 2011;2(2):72-6.10.4103/2229-3485.80371 


\section{Clinical ethics}

16 Reitsma AM, Moreno JD. Surgical research, an elusive entity. Am J Bioeth 2003:3(4):49-50.

17 Holtedahl R, Brox JI, Tjomsland O. Placebo effects in trials evaluating 12 selected minimally invasive interventions: a systematic review and meta-analysis. BMJ Open 2015;5(1):e007331.

18 Degen RM, Lebedeva Y, Birmingham TB, et al. Trends in knee arthroscopy utilization: a gap in knowledge translation. Knee Surg Sports Traumatol Arthrosc 2019;392(10160):1-9.

19 Pronovost PJ, Berenholtz SM, Needham DM. Translating evidence into practice: a model for large scale knowledge translation. BMJ 2008;337:a1714.
20 Brownlee S, Chalkidou K, Doust J, et al. Evidence for overuse of medical services around the world. The Lancet 2017;390(10090):156-68.

21 Davis Det al. The case for knowledge translation: shortening the journey from evidence to effect. BMJ 2003;327(7405):33-5.

22 Bunzli S, Dowsey MM, Choong PF. Sham surgery: justified but practical? A systematic review of sham surgery trials in orthopaedics. SA Orthopaedic Journal 2018:17(3):14-24.

23 Savulescu J, Wartolowska K, Carr A. Randomised placebo-controlled trials of surgery: ethical analysis and guidelines. J Med Ethics 2016;42(12):776-83. 